\title{
Holographic fabrication of diamondlike photonic crystal template using two-dimensional diffractive optical elements
}

\author{
Di Xu, ${ }^{1}$ Kevin P. Chen, ${ }^{1, a)}$ Kris Ohlinger, ${ }^{2}$ and Yuankun Lin ${ }^{2}$ \\ ${ }^{1}$ Department of Electrical and Computer Engineering, University of Pittsburgh, Pittsburgh, \\ Philadelphia 15261, USA \\ ${ }^{2}$ Department of Physics and Geology, University of Texas-Pan American, Edinburg, Texas 78541, USA
}

(Received 3 April 2008; accepted 4 May 2008; published online 21 July 2008)

\begin{abstract}
This letter demonstrates holographic fabrication of three-dimensional diamondlike photonic crystal templates in SU8 photoresist using a single diffractive optical element. Five coherent laser beams produced by a two-dimensional phase mask were used to construct face-centered-cubic or tetragonal interference patterns. The superposition of two interference patterns through double exposures yields diamondlike photonic crystal templates in SU8. Photonic bandgap calculation reveals a full bandgap in inverse structures based on the template. The utilization of the two-dimensional phase mask simplifies the fabrication configuration in multiple beam holographic lithography for three-dimensional photonic fabrication. (C) 2008 American Institute of Physics.
\end{abstract}

[DOI: $10.1063 / 1.2959092]$

Three-dimensional (3D) photonic crystal $(\mathrm{PhC})$ has attracted enormous interests in the last decade in both science and technology communities. Its unique capability to trap photons offers an interesting scientific perspective and can be useful for optical communication and sensing. ${ }^{1,2}$ However, the fabrication of large-scale 3D photonic structures at submicron scale remains a great challenge. Considerable efforts have been dedicated to develop fabrication techniques to produce large area defect-free 3D photonic structures toward device applications. These include layerby-layer lithography, ${ }^{3}$ self-assembly, ${ }^{4}$ direct laser writing, ${ }^{5}$ and holographic lithography techniques. ${ }^{6}$

Among all fabrication approaches, holographic lithography has been recognized as a primary method to produce $3 \mathrm{D}$ structures due to its controllability, scalability, and flexibility. All fourteen Bravais periodic lattices can be fabricated using multiple-beam holographic fabrication. ${ }^{7,8}$ In addition, the holographic lithography enables the fabrication of complex 3D photonic structures such as a diamondlike structure with large bandgap. ${ }^{9}$ Traditionally, multiple coherent laser beams needed to produce holographic interference patterns were constructed by bulk optical elements such as beamsplitters and mirrors. ${ }^{10}$ It involves delicate optical alignments, large footprint for optical setup, and stable mechanical platforms. To simplify the construction of multiple coherent beams, both refractive and diffractive optical elements have been recently adapted to construct multiple-beam interference patterns for 3D PhC fabrications. ${ }^{11,12}$ These approaches replace all beam-forming optical components by a single optical element such as a phase mask. The phase mask approach offers a wafer-scale fabrication approach to produce 3D nanooptical patterns, which can be incorporated into existing photolithography process for photonic device chip fabrication. The flexibility of one-dimensional (1D) phase mask approaches for $\mathrm{PhC}$ fabrications have been demonstrated in the fabrication of woodpile, orthorhombic, and tetragonal structures. ${ }^{11}$

In this letter we extended the phase-mask approach for holographic fabrication from 1D phase mask to two-

\footnotetext{
${ }^{a)}$ Electronic mail: kchen@engr.pitt.edu.
}

dimensional (2D) phase mask. The flexibility of 2D phase mask design enables the construction of multiple-beam interference patterns for the fabrication of complex 3D periodic structure such as diamondlike structures. ${ }^{13}$ Diamondlike $\mathrm{PhC}$ structures possess the largest photonic bandgap among all possible PhCs. However, the complex structures pose a great fabrication challenge. In this letter, we demonstrate this flexibility by fabricating diamondlike $\mathrm{PhC}$ structures in SU8 photoresist.

The 2D phase masks used in this experiment were fabricated in a thin negative-tone SU8-2035 photoresist (MicroChem Corp.). The photoresist was spin coated on a glass substrate with a thickness of $2 \mu \mathrm{m}$. After spin coating, the sample was prebaked at $65^{\circ} \mathrm{C}$ for $5 \mathrm{~min}$ and $95^{\circ} \mathrm{C}$ for $3 \mathrm{~min}$ before receiving the laser exposure. The source beam from an Argon Ion laser was collimated and split into two coherent linearly polarized beams with equal intensity using a beam splitter. Two laser beams were recombined by a pair of mirrors and overlapped across the photoresist with a certain angle to produce an interference pattern. The polarizations of two interference beams are parallel to the surface of the photoresist. The photoresist solution was sensitized for $514.5 \mathrm{~nm}$ laser wavelength by adding $0.25 \mathrm{wt} \% 5,7$ diiodo-3-butoxy-6-fluorone (H-Nu470) and $2.5 \mathrm{wt} \%$ (4octyloxphenyl) phenyl iodonium hexafluoroantimonate (OPPI) photoinitiators (Spectra Group Limited). ${ }^{12}$ After the first exposure, the sample stage was rotated along the normal direction of the sample by angle $\alpha$ and received a second exposure. Then the samples were postbaked at $65^{\circ} \mathrm{C}$ for $10 \mathrm{~min}$ and $95^{\circ} \mathrm{C}$ for $5 \mathrm{~min}$ followed by photoresist development for $5 \mathrm{~min}$. After that, the phase mask was hardened by baking at $120^{\circ} \mathrm{C}$ for $10 \mathrm{~min}$. Figures 1 (a) and 1(b) show the scanning electronic micrograph (SEM) and simulated surface topography in SU8 resulting from double laser exposures with $90^{\circ}$ rotation angle between exposures. Figure 1(c) shows the diffraction pattern produced by the phase mask.

To produce more complex multiple-beam interference patterns, multiple laser exposures can be used to create a 2D phase mask. Figures 1(d) and 1(e) show SEM and simulated topography resulting from three laser exposures; the sample was rotated by $\alpha=60^{\circ}$ each time between exposures. The 

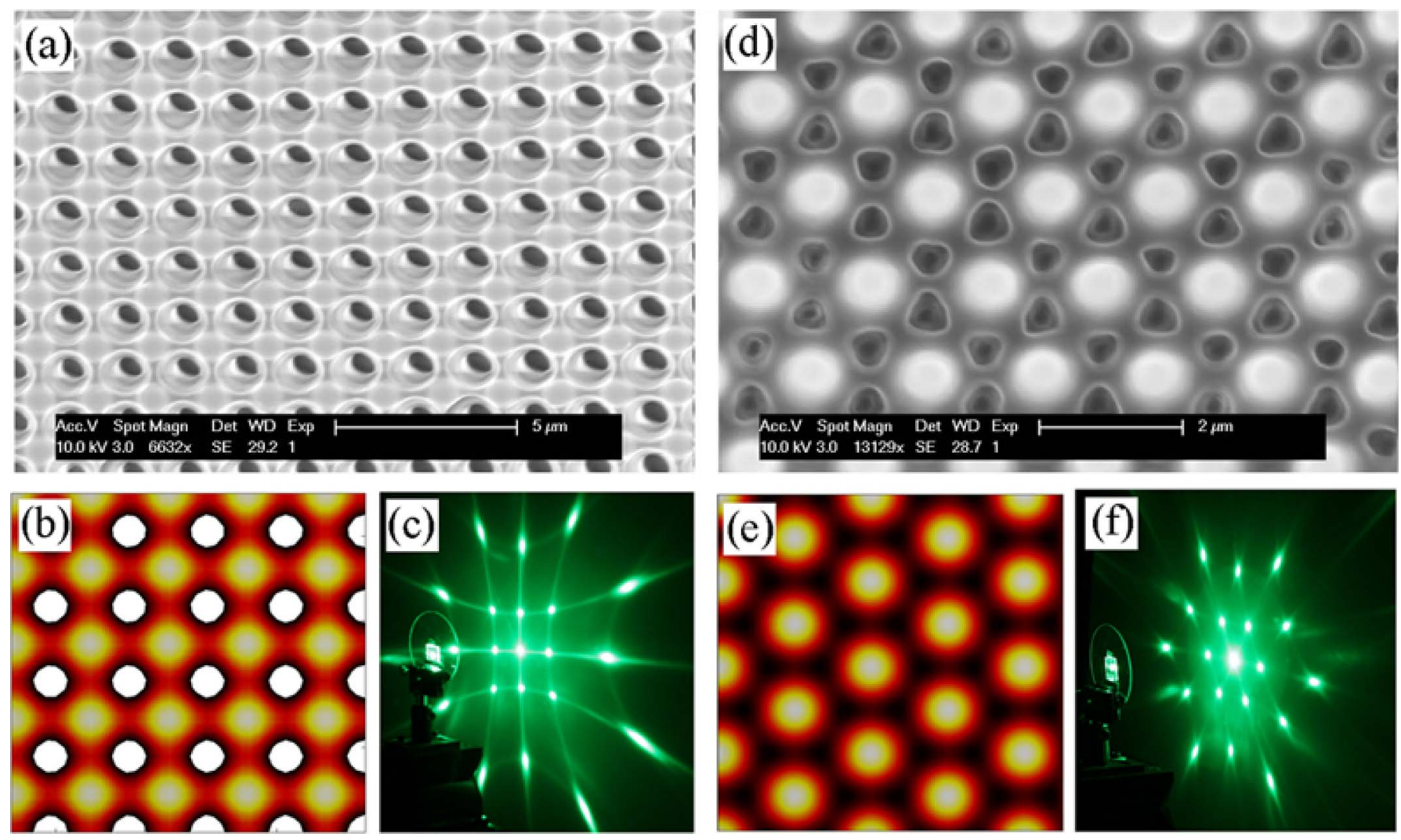

FIG. 1. (Color online) The SEM, simulated surface and diffractive pattern of 2D phase masks made by double exposures with rotation angle $\alpha=90^{0}[(\mathrm{a})-(\mathrm{c})]$ and by triple exposures with $\alpha=60^{\circ}[(\mathrm{d})-(\mathrm{f})]$.

diffraction pattern produced by this phase mask is shown in Fig. 1(f). Its interference pattern produces a face-centered hexagonal structure.

The 2D phase mask we used for $\mathrm{PhC}$ fabrication has typically a period of $1.1 \mu \mathrm{m}$. It has an orthogonal surface profile $\left(\alpha=90^{\circ}\right)$ and its laser beams diffraction is schematically shown in Fig. 2(a). The diffraction angle and diffraction efficiency of four first-order diffraction beams labeled as $(1,0),(-1,0),(0,1),(0,-1)$ were measured to be $20^{\circ}$ and $10 \%$, respectively. The four second-order diffraction beams $(1,1),(1,-1),(-1,1),(-1,-1)$ had much lower diffraction efficiency at $1.5 \%$. The third and higher order beams have negligible intensities. Therefore, only the zeroth and first order beams were used to determine crystal structures based on
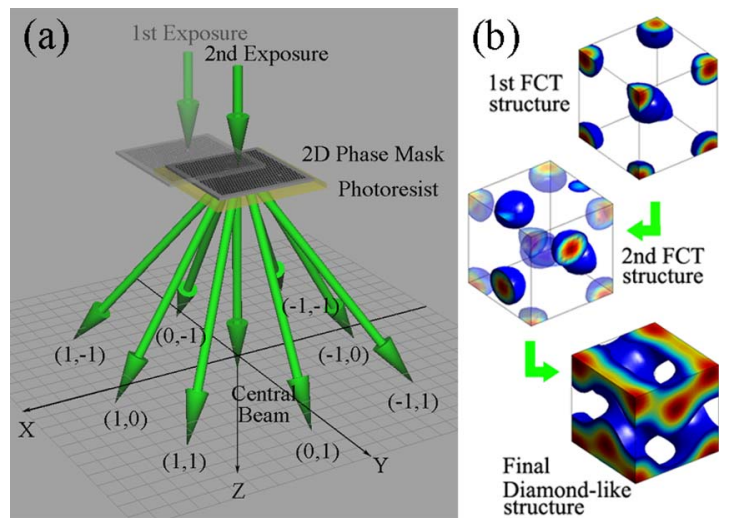

FIG. 2. (Color online) (a) Sketch of propagation of light through an orthogonal 2D phase mask. FCT pattern is generated by recording the intensity distribution of multiple-beam interference in SU8 photoresist. (b) Diamondlike structure constructed by double exposures with one FCT pattern shifted by $\Delta x=0.5 a$ and $\Delta z=0.25 c$. isointensity surface of the interference pattern. ${ }^{14}$ The interference of five beams produced by the 2D phase mask yields a face-centered-cubic or face-centered-tetragonal (FCT) structure.

Figure 2(b) illustrates the path for lattice translation from FCT to diamondlike structures. Theoretically, the diamondlike structures can be viewed as the superposition of two FCT structures with a lattice displacement. ${ }^{14-16}$ This can be achieved by double exposures through the $2 \mathrm{D}$ phase mask on the same photoresist sample with phase mask displacement along the [201] direction in a Cartesian coordinator system for a quarter diagonal length between exposures. The offsets $\Delta x$ and $\Delta z$ between two exposure patterns inferred from simulation are $0.5 a$ and $0.25 c$, respectively, where $a$ and $c$ are the lattice constants of the FCT structure in $x$ and $z$ directions. These actual precise displacements were performed by three-axis high-precision Newport motorized linear stages (PM500-4L). The simulated isointensity surfaces of the first FCT, second shifted FCT, and final superimposed structures are shown in Fig. 2(b).

The 3D template was fabricated in a thick SU8 film sample of $20 \mu \mathrm{m}$. The concentration of $\mathrm{H}-\mathrm{Nu} 470$ in photoresist solution was increased to $0.5 \mathrm{wt} \%$ to accelerate the photoresist cross linking. $20 \mathrm{M} \%$ of Triethylamine was added into the recipe to increase the contrast ratio of the template during the photoresist development. ${ }^{10}$ To avoid reaching the boiling point of Triethylamine of $89.7^{\circ} \mathrm{C}$, the prebake procedure was adjusted to one step at $65^{\circ} \mathrm{C}$ for $20 \mathrm{~min}$. The exposure laser intensity was set at $100 \mathrm{~mW} / \mathrm{cm}^{2}$ for both laser exposures. Figures 3(a) and 3(b) show SEM topview of diamondlike structures recorded in SU8. The surface feature is consistent with the simulation results of the (001) plane of a diamondlike structure in both inset of Figs. 

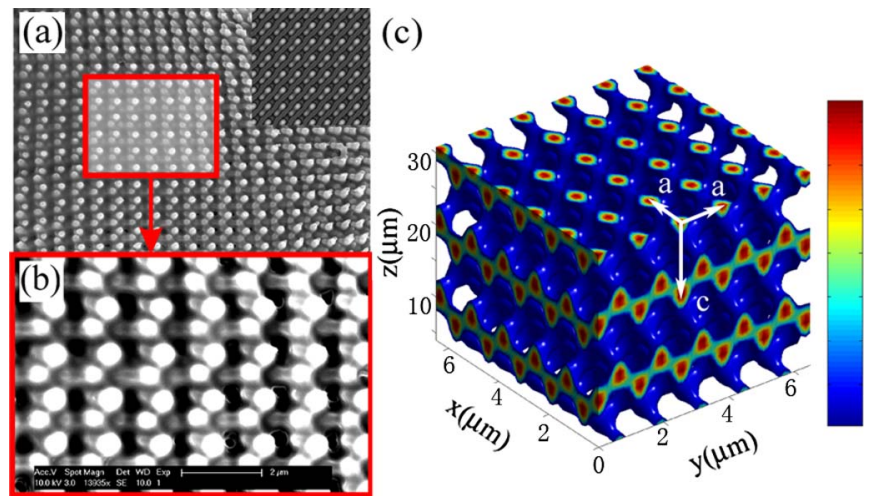

FIG. 3. (Color online) (a) SEM top view of the fabricated structure together with inserted surface simulation based on FCT pattern superposition. (b) Enlarged view of the fabricated structure. (c) Simulated interconnected 3D diamondlike structures through double exposures.

3(a) and 3(c). The cross linking between two FCT structures formed by two laser exposures produces a stable 3D template for further inversion processes to create high-index contrast structures.

The photonic bandgap for the silicon inverse structures is calculated and shown in Fig. 4 using the MIT PHOTONICBANDS Package. ${ }^{17}$ Figure 4(a) shows the photonic band structure for $c / a=1.5$ for the silicon inverse structure where the dielectric constant of 11.9 is used for silicon. ${ }^{18}$ The optimal silicon filling fraction for diamondlike structure is approximately $18.4 \%$ while $20.5 \%$ for a FCT structure. The band structure shows that a full photonic bandgap of $27 \%$ of the gap center frequency exists between the second and third bands of the optimal diamondlike structure. This is in con-

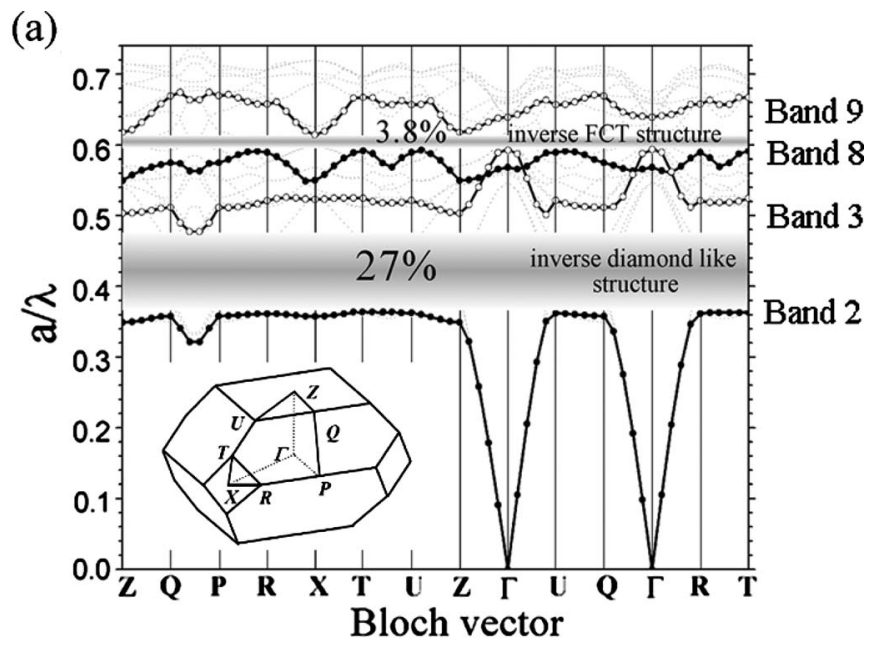

(b)

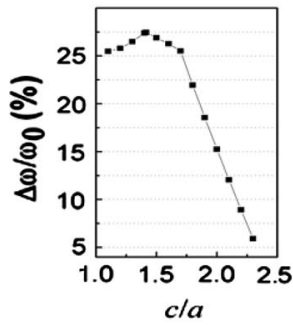

(c)

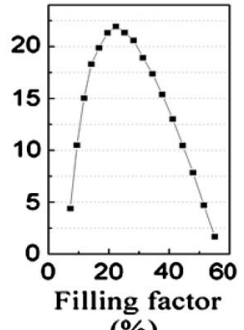

(\%) (d)

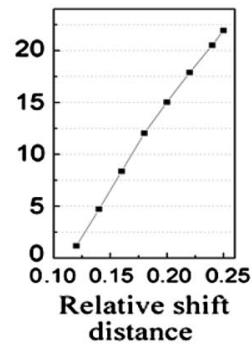

FIG. 4. (a) Photonic band diagram for the inverse FCT structure and FCT based diamondlike structure. The inset is the first Brillouin surface. [(b)-(d)] are the gap size as a function of c/a ratio, dielectric filling factor, and relative shift distance, respectively. trast to the maximal photonic bandgap of $3.8 \%$ between the eighth and ninth bands of a single FCT inverse structure with the same $c / a$ value. The gap size is increased by the operation of constructing the diamondlike lattice formed by double laser exposures.

The bandgap of the fabricated diamondlike structure depends on $c / a$ ratio (the diffractive angle), the filling ratio of the structure (exposure dose), and the relative lattice shift distance. The bandgap size dependence on these fabrication parameters are shown in Figs. 4(b)-4(d). Figure 4(b) indicates a need of larger diffraction angle or a close match between lattice constant $c$ and $a$. A $c / a$ ratio from 1.0 to 1.7 will maintain a bandgap larger than $20 \%$. The bandgap is closed when the $c / a$ is greater than 2.5. In addition, the filling fraction of the structure also impacts the bandgap, which can be controlled by laser exposure dosage and the photoresist development time. Figure 4(c) shows this trend for a diamondlike structure with $c / a=1.8$. The optimal dielectric filling fraction was found to be $23 \%$ for the inverse structure. In Fig. 4(d) the bandgap size is shown as a function of phase mask translation distance between two exposures. When the second FCT lattice is shifted away the optimum location of $(0.5 a, 0,0.25 c)$ toward $(0,0,0)$, for the inverse structure with $c / a=1.8$, the bandgap size decreases almost linearly during the transition from the diamondlike structure to the FCT structure.

In summary, we have demonstrated a $2 \mathrm{D}$ phase mask approach to generate multicoherent beams for complex $\mathrm{PhC}$ fabrications. Diamondlike PhC templates were fabricated in SU8 using this approach. The utilization of the 2D phase mask simplifies the fabrication configuration in multiple beam holographic lithography for complex 3D optical fabrication.

This work was supported by research grants from the U.S. National Science Foundation CMMI-0609345 (Y.L.) and CMMI-0556086 (K.P.C.), and the U.S. Air Force.

${ }^{1}$ S. John, Phys. Rev. Lett. 58, 2486 (1987).

${ }^{2}$ E. Yablonovitch, Phys. Rev. Lett. 58, 2059 (1987).

${ }^{3}$ K. M. Ho, C. T. Chan, C. M. Soukoulis, R. Biswas, and M. Sigalas, Solid State Commun. 89, 413 (1994).

${ }^{4}$ S. H. Park, D. Qin, and Y. Xia, Adv. Mater. (Weinheim, Ger.) 10, 1028 (1998).

${ }^{5}$ M. Deubel, G. V. Freymann, M. Wegener, S. Pereira, K. Busch, and C. M. Soukoulis, Nat. Mater. 3, 444 (2004).

${ }^{6}$ M. Campbell, D. N. Sharp, M. T. Harrison, R. G. Denning, and A. J. Turberfield, Nature (London) 404, 53 (2000).

${ }^{7}$ L. Z. Cai, X. L. Yang, and Y. R. Wang, Opt. Lett. 27, 900 (2002).

${ }^{8}$ V. Berger, O. Gauthier-Lafaye, and E. Costard, J. Appl. Phys. 82, 60 (1997).

${ }^{9}$ D. N. Sharp, A. J. Turberfield, and R. G. Denning, Phys. Rev. B 68, 205102 (2003).

${ }^{10}$ S. Yang, M. Megens, J. Aizenberg, P. Wiltzius, P. M. Chaikin, and W. B. Russel, Chem. Mater. 14, 2831 (2002).

${ }^{11}$ Y. K. Lin, D. Rivera, and K. P. Chen, Opt. Express 14, 887 (2006).

${ }^{12}$ Z. Poole, D. Xu, K. P. Chen, I. Olvera, K. Ohlinger, and Y. Lin, Appl. Phys. Lett. 91, 251101 (2007).

${ }^{13}$ Y. Lin, P. R. Herman, and E. L. Abolghasemi, J. Appl. Phys. 97, 096102 (2005).

${ }^{14}$ O. Toader, T. Y. M. Chan, and S. John, Phys. Rev. Lett. 92, 043905 (2004).

${ }^{15}$ Y. Lin, D. Rivera, Z. Poole, and K. P. Chen, Appl. Opt. 45, 7971 (2006).

${ }^{16}$ J. H. Moon, S. Yang, D. J. Pine, and S. M. Yang, Opt. Express 13, 9841 (2005).

${ }^{17}$ S. G. Johnson and J. D. Joannopoulos, Opt. Express 8, 173 (2001).

${ }^{18}$ N. Tétreault, G. V. Freymann, M. Deubel, M. Hermatschweiler, F. P. Willard, S. John, M. Wegener, and G. A. Ozin, Adv. Mater. (Weinheim, Ger.) 18, 457 (2006). 
Applied Physics Letters is copyrighted by the American Institute of Physics (AIP). Redistribution of journal material is subject to the AIP online journal license and/or AIP copyright. For more information, see http://ojps.aip.org/aplo/aplcr.jsp 